\title{
Deposição de calda de pulverização e produtividade da soja cultivada em diferentes arranjos espaciais
}

\section{Deposition of spray solution and productivity of soybean cultivated in different spatial arrangements}

\author{
Vandoir Holtz $^{\mathrm{I}^{*}}$ Rodney Ferreira Couto ${ }^{\mathrm{I}}$ Danilo Gomes de Oliveira ${ }^{\mathrm{I}}$ \\ Elton Fialho dos Reis ${ }^{\mathrm{I}}$
}

\section{RESUMO}

O principal objetivo da aplicação de produtos fitossanitários é o controle eficiente de insetos-praga, patógenos e plantas daninhas. $O$ arranjo espacial da cultura pode interferir na estrutura morfológica da planta e alterar a arquitetura da planta, criando condições diferenciadas para a penetração das gotas no dossel. Este trabalho teve como objetivo avaliar o efeito de diferentes estandes e espaçamentos entrelinhas na deposição de calda de pulverização e na produtividade da cultura da soja. Foi utilizado um delineamento experimental em blocos casualizados no esquema fatorial $2 x 6$, sendo dois estandes (160.000 e 240.000 plantas $\left.\mathrm{ha}^{-1}\right)$ e seis espaçamentos entrelinhas $(0,30 ; 0,40 ; 0,50$; 0,$60 ; 0,70 \mathrm{~m}$ e 0,50m em semeadura com linhas cruzadas), com quatro repetições. Foram avaliados o tempo de fechamento das entrelinhas, a porcentagem de fechamento das entrelinhas, a deposição de calda de pulverização nos terços superior, médio $e$ inferior da planta, a razão deposição entre o terço superior e inferior do dossel e a produtividade. Os tratamentos com espaçamentos de 0,6 e 0,7m não atingiram 100\% de fechamento. Houve maior deposição no terço inferior nas parcelas semeadas em linhas cruzadas. A maior produtividade de grãos ocorreu no espaçamento de $0,4 \mathrm{~m}$. Os resultados indicam que a alteração do arranjo espacial pode aumentar o tempo de fechamento e/ou reduzir o fechamento total das entrelinhas da soja, possibilitando controle eficiente das doenças de baixeiro.

Palavras-chave: tecnologia de aplicação, população de plantas, espaçamento, Glycine max.

\section{ABSTRACT}

The main purpose of the application of pesticides is the efficient control of insects-pests, diseases and weeds. The spatial arrangement of culture can interfere in the structure of the plant and change plant architecture, creating differentiated conditions for the penetration of the droplets in the canopy. This study aimed to evaluate the effect of different population of plants and row spacing in the deposition of spray solution and productivity of the soybean. It was used a randomized block design in factorial $2 \times 6$, with two population of plants (160.000 and 240.000 plants $\mathrm{ha}^{-1}$ ) and six row spacings $(0.30 ; 0.40 ; 0.50 ; 0.60 ; 0.70 \mathrm{~m}$ and $0.50 \mathrm{~m}$ with sowing in crossed lines), with four blocks. It was evaluated time to canopy closure, the percentage of canopy closure, the deposition of spray solution in the upper, middle and lower plant, the ratio of deposition between the upper and lower third of the plant and the crop yield. Treatments with spacings of 0.6 and $0.7 \mathrm{~m}$ did not reach $100 \%$ closure. There was a higher deposition in the lower third in the plots sown in lines cross. The highest grain yield occurred at a spacing of $0.4 \mathrm{~m}$. The results indicate that the change in the spatial arrangement can increase the time of closing and/ or reduce the total closure of the soybean lines, enabling efficient control of diseases in the plant bottom.

Keywords: application technology, plant populations, spacing, Glycine max.

\section{INTRODUÇÃO}

O grande desafio a ser vencido pela tecnologia de aplicação na cultura da soja consiste em fazer com que as gotas atravessem a camada superior de folhas das plantas e cheguem com condições de se distribuir, depositar e cobrir, adequadamente, todo o dossel (FERREIRA \& OLIVEIRA, 2008).

A planta de soja é dotada de grande plasticidade morfológica (MUNDSTOCK \& THOMAS, 2005). Devido a essa plasticidade, a soja apresenta capacidade de adaptação às diferentes condições ambientais e de manejo, por meio de modificações morfológicas, da arquitetura e dos

'Departamento de Engenharia Agrícola, Universidade Estadual de Goiás (UEG), Campus Henrique Santillo, BR 153, n 3.105, Fazenda Barreiro do Meio, 75132-903, Anápolis, GO, Brasil. E mail: vandoirholtz@hotmail.com.*Autor para correspondência.

$$
\begin{array}{cc}
\text { Recebido 04.06.13 Aprovado 29.01.14 Devolvido pelo autor 05.06.14 } \\
\text { CR-2013-0783.R1 }
\end{array}
$$


componentes do rendimento. Essas modificações podem estar relacionadas com a fertilidade do solo, população de plantas e espaçamento entrelinhas (PIRES et al., 2000; RAMBO et al., 2003).

Arranjo espacial é uma prática de manejo importante que pode melhorar a interceptação da radiação por meio da cobertura do solo mais completo (HEITHOLT et al., 2005), contudo, o estreitamento das entrelinhas pode estabelecer características diferenciadas do ponto de vista da patogênese, fisiologia da planta e tecnologia de aplicação (MADALOSSO et al., 2010). Além disso, características fisiológicas do terço médio e inferior podem estar sendo suprimidas ou pouco exploradas agronomicamente, devido ao arranjo de plantas inadequado à cultivar (TAIZ \& ZEIGER, 2004).

De acordo com PHIPPS et al. (2006), o arranjo espacial das plantas e uma estratégia de aplicação que produza maior cobertura foliar e penetração por aplicação de fungicida, também produzirá o melhor resultado.

Em razão disso, o objetivo deste trabalho foi avaliar a deposição de calda de pulverização em diferentes estandes e espaçamentos entrelinhas na cultura da soja, sob a hipótese de que é possível melhorar a deposição por meio de modificação do arranjo espacial da cultura.

\section{MATERIAL E MÉTODOS}

O trabalho foi realizado na Estação Experimental de Anápolis-GO da Agência Goiana de Assistência Técnica, Extensão Rural e Pesquisa Agropecuária-EMATER, com latitude Sul de $16^{\circ} 19^{\prime} 48^{\prime \prime}$, longitude Oeste de 48 $18^{\prime} 23^{\prime \prime}$ e altitude média de $1050 \mathrm{~m}$. O clima desta região é do tipo AW tropical úmido, segundo a classificação de Köeppen, caracterizado pelo inverno seco e o verão chuvoso. O solo do local do experimento é classificado como Latossolo Vermelho distrófico, textura argilosa, com inclinação média de 4\%.

O delineamento experimental empregado foi em blocos casualizados, em esquema fatorial $2 \times 6$, com quatro blocos, totalizando 48 parcelas experimentais. Cada bloco foi composto por doze parcelas constituídas por linhas de cultivo de $5,0 \mathrm{~m}$ de comprimento no mesmo sentido ao bloco, e $3,0 \mathrm{~m}$ de largura.

Foram avaliados os estandes de 160.000 e 240.000 plantas ha $\mathrm{h}^{-1}$, nos espaçamentos entrelinhas de 0,$30 ; 0,40 ; 0,50 ; 0,60 ; 0,70 \mathrm{~m}$ e a semeadura com linhas cruzadas, espaçamento entrelinhas de $0,50 \mathrm{~m}$, tanto na longitudinal como na perpendicular. A cultivar de soja utilizada foi a 'BRS Valiosa RR', geneticamente modificada, desenvolvida pelo Convênio Cerrados (Embrapa/Agência Rural/CTPA).

A área experimental foi preparada pelo sistema convencional e a semeadura foi realizada no dia 14 de novembro de 2011. As sementes foram tratadas com inseticida friponil na dose de $200 \mathrm{~mL}$ por $100 \mathrm{~kg}$ de sementes, fungicida carboxina + tiram na dose de $250 \mathrm{~mL}$ para $100 \mathrm{~kg}$ de sementes e inoculadas com bactéria do gênero Bradyhizobium na dose de $150 \mathrm{~mL}$ do produto comercial para $50 \mathrm{~kg}$ de sementes. Como adubação, foram aplicados $450 \mathrm{~kg} \mathrm{ha}^{-1} \mathrm{da}$ fórmula NPK 08-20-20 + 50 $\mathrm{kg} \mathrm{ha}^{-1}$ de FTE, conforme recomendação. A colheita das parcelas foi realizada no final do mês abril de 2012.

O tempo de fechamento foi obtido pela contagem em dias, correspondendo ao período necessário para que ocorresse $100 \%$ do fechamento das entrelinhas. A porcentagem de fechamento foi quantificada nas parcelas que não tiveram total fechamento, por meio de imagens digitais, obtidas de forma perpendicular à superfície do solo, com máquina fotográfica marca Sony, modelo Cyber-Shot, com resolução de 7,2 megapixels. A área não coberta pelas folhas foi identificada com a utilização do programa computacional "AFSoft", desenvolvido e disponibilizado pela EMBRAPA(2012) e o percentual de fechamento com o programa computacional de processamento e análise de imagem em Java, “ImageJ" (NIH, 2012).

Para a aplicação da calda de pulverização, foi utilizada uma barra de aplicação pressurizada com $\mathrm{CO}_{2}$, munida de quatro bicos espaçados entre si de $0,5 \mathrm{~m}$, com ponta de aplicação do tipo jato plano defletor, modelo TT11002 da marca Teejet, que gerou gotas com DMV de $211,17 \mu \mathrm{m}$ e $11,33 \%$ de gotas menores que $100 \mu \mathrm{m}$. O volume aplicado foi de $180 \mathrm{~L}$ $\mathrm{ha}^{-1}$, à pressão de $320 \mathrm{kPa}$, com velocidade média de aplicação de $5,7 \mathrm{~km} \mathrm{~h}^{-1}$. A altura de trabalho em relação à cultura foi de $0,50 \mathrm{~m}$. A aplicação da calda de pulverização foi realizada após a avaliação da porcentagem de fechamento, quando a cultura estava no estádio fenológico definido como R2 e as plantas apresentavam altura média de $0,70 \mathrm{~m}$ e índice de área foliar (IAF) médio de 3,9. Durante a aplicação, a temperatura média foi de $28,76^{\circ} \mathrm{C}$, a umidade relativa do ar média de $43,80 \%$ e velocidade média do vento de $1,34 \mathrm{~m} \mathrm{~s}^{-1}$.

O estudo de deposição da calda pulverizada foi feito por meio da quantificação de um traçador. A solução traçadora foi constituída de água e corante alimentício Azul Brilhante (Corante Azul FCF, Duas Rodas Industrial), catalogado internacionalmente 
pela Food, Drug \& Cosmetic como FD\&C Blue n. 1 , na dose de $0,340 \mathrm{~kg} \mathrm{ha}^{-1}$, conforme metodologia adaptada de PALLADINI et al. (2005). Para isso, foi usado um espectrofotômetro da marca Bioespectro, modelo SP-220, com lâmpada de tungstênio, com vapor de halogênio e cubetas de vidro com caminho óptico de $10 \mathrm{~mm}$, no comprimento de onda de $630 \mathrm{~nm}$ para o azul brilhante.

Após a aplicação, foram coletadas duas folhas em cada terço, em cinco plantas por parcela. Depois da remoção do depósito, as folhas foram fotografadas e sua área foliar quantificada utilizando o programa computacional "ImageJ" (NIH, 2012).

Para obtenção do depósito na planta, foi feita a curva de calibração do espectrofotômetro, a partir de soluções com concentrações conhecidas do corante, que gerou uma equação de regressão $\mathrm{y}=0,5798 \mathrm{x}-0,0017 \quad\left(\mathrm{R}^{2}=0,99\right)$. Os dados de absorbância foram transformados em volume $(\mu \mathrm{L})$. Procedeu-se, então, à divisão do volume depositado pela área das folhas, obtendo-se, assim, a quantidade em $\mu \mathrm{L} \mathrm{cm}^{-2}$. Também foi calculada a razão de deposição entre os volumes retidos no terço superior e inferior.

Para a produtividade de grãos de soja, toda a área útil de cada parcela foi colhida manualmente $\mathrm{e}$ após trilhada, seus grãos foram pesados e determinada a sua umidade. Posteriormente, o valor da massa obtida foi transformado para $\mathrm{kg} \mathrm{ha}^{-1}$, com sua umidade corrigida para $13 \%$ em base úmida.

Os dados foram submetidos ao teste de Hartley para verificar a condição de homocedasticidade e, após, foi realizada a análise de variância, aplicandose o teste F, a 5\% de probabilidade. Quando significativos, suas médias foram comparadas pelo Teste de Tukey. Para as análises estatísticas, foi utilizado o programa computacional SISVAR 5.3 (FERREIRA, 2011).

\section{RESULTADOS E DISCUSSÃO}

A análise dos dados possibilitou verificar um comportamento diferenciado da cultura da soja nos arranjos espaciais e em relação à tecnologia de aplicação sobre o dossel.

Para a análise de variância do tempo de fechamento das entrelinhas, foram utilizados somente os tratamentos em que todas as parcelas tiveram $100 \%$ de fechamento, o que ocorreu nos tratamentos com os espaçamentos de 0,$3 ; 0,4 ; 0,5 \mathrm{~m}$; e $0,5 \mathrm{~m}$ em linhas cruzadas. O tempo de fechamento apresentou diferenças significativas para o estande, bem como para o espaçamento, mas não foi verificada a interação entre os fatores.

Tanto o tempo de fechamento, como a porcentagem de fechamento das entrelinhas foram comprometidos pelo aumento do espaçamento (Tabela 1). Esses resultados concordam com os

Tabela 1-Teste de média e síntese da análise de variância (quadrado médio) do tempo de fechamento e porcentagem de fechamento das entrelinhas da cultura da soja para os estandes de 160000 e 240000 plantas ha ${ }^{-1}$ nos espaçamentos entrelinhas de 0,$30 ; 0,40 ; 0,50$; 0,$60 ; 0,70 \mathrm{~m}$ e semeadura em linhas cruzadas $(0,5 \times 0,5) \mathrm{m}$.

\begin{tabular}{llc}
\hline & Tempo de fechamento (dias) & Porcentagem de fechamento (\%) \\
\hline Espaçamento & $55,75 \mathrm{~b}$ & $100 \mathrm{a}$ \\
$0,30 \mathrm{~m}$ & $58,50 \mathrm{ab}$ & $100 \mathrm{a}$ \\
$0,40 \mathrm{~m}$ & $62,25 \mathrm{a}$ & $100 \mathrm{a}$ \\
$0,50 \mathrm{~m}$ & $59,50 \mathrm{ab}$ & $100 \mathrm{a}$ \\
$0,5 \mathrm{x} 0,5$ & s.f. & $99,21 \mathrm{a}$ \\
$0,60 \mathrm{~m}$ & s.f. & $96,85 \mathrm{~b}$ \\
$0,70 \mathrm{~m}$ & & \\
Estandes & $60,56 \mathrm{a}$ & $99,41 \mathrm{a}$ \\
160000 plantas ha ${ }^{-1}$ & $57,43 \mathrm{~b}$ & $99,34 \mathrm{a}$ \\
240000 plantas ha ${ }^{-1}$ & $78,12^{* *}$ & $0,23^{\mathrm{NS}}$ \\
Estande & $57,67 * *$ & $12,73^{* *}$ \\
Espaçamento & 15,46 & 0,91 \\
Estande x Espaçamento & 12,61 & 1,83 \\
Resíduo & 6,02 & 1,36 \\
Coeficiente de variação $(\%)$ & & \\
\hline
\end{tabular}

Médias seguidas de mesma letra minúscula na coluna não diferem estatisticamente pelo teste de Tukey a 5\% de probabilidade. Ns. não significativo $(\mathrm{P}>0,05) ;{ }^{*}$ Significativo a $5 \%$ de probabilidade pelo teste $\mathrm{F}$; **Significativo a $1 \%$ de probabilidade pelo teste $\mathrm{F}$; s.f.: não atingiu $100 \%$ de fechamento em todas as parcelas. 
HEIFFIG et al. (2006) nos espaçamentos de 0,60 e $0,70 \mathrm{~m}$, KNEBEL et al. (2006) no de $0,675 \mathrm{~m}$ e HEITHOLT et al. (2005) no de $0,71 \mathrm{~m}$, em que o fechamento da entrelinha não atingiu $100 \%$.

A análise de variância para a deposição de calda mostrou influência significativa para a fonte de variação espaçamento no terço inferior. A maior quantidade de calda depositada no terço inferior foi encontrada na soja cultivada em semeadura em linhas cruzadas, apresentando maior valor comparado ao espaçamento de $0,30 \mathrm{~m}$. Esse resultado é indicativo de que a alteração no arranjo espacial provoca mudanças na arquitetura das plantas, que foram favoráveis à técnica utilizada na aplicação. Dessa forma, a escolha do arranjo espacial pode ser feita para privilegiar a técnica de aplicação e melhorar a distribuição da calda no dossel. A razão de deposição entre o terço superior e inferior do dossel apresentou diferenças significativas em função do espaçamento. A menor razão de deposição foi encontrada para a semeadura em linhas cruzadas, que diferiu estatisticamente do espaçamento $0,30 \mathrm{~m}$. A razão de deposição entre o terço superior e inferior é um índice que expressa a diferença na cobertura entre os terços do dossel da cultura. Menor razão indica melhor distribuição de calda de pulverização ao longo da planta.

A menor razão de deposição pode ocorrer devido a uma menor deposição no terço superior ou a uma maior deposição no terço inferior, bem como aos dois fatores. No presente estudo, não foram constatadas diferenças significativa da deposição no terço superior, portanto a menor razão de deposição ocorreu pela maior deposição no terço inferior.

Os resultados da deposição e razão de deposição estão apresentados na tabela 2, em que, além das diferenças estatísticas, é possível observar que as médias da deposição seguem uma tendência de maior depósito nos maiores espaçamentos, acompanhada de menor razão de deposição, com destaque para a relação entre a deposição no terço inferior no espaçamento de $0,70 \mathrm{~m}$, que foi $59,03 \%$ maior em relação ao espaçamento de $0,30 \mathrm{~m}$.

Esses resultados corroboram os de MADALOSSO et al. (2010), que, em estudo específico com ferrugem asiática, verificaram que o espaçamento das entrelinhas de $0,60 \mathrm{~m}$ proporcionou menores valores da ferrugem asiática acumulada, atribuindo este resultado ao fato de que o aumento do espaçamento das entrelinhas otimiza a eficiência dos programas de controle sobre o alvo, explicado pela maior exposição das folhas do terço médio e inferior à penetração e cobertura de gotas pulverizadas. De modo semelhante, FERREIRA (2012), avaliando aplicações realizadas antes do fechamento na cultura da soja, concluiu que a aplicação neste momento encontra condições mais favoráveis para a penetração, com aumento da taxa de deposição no terço inferior. Já DEBORTOLI et al. (2012) afirmaram que há

Tabela 2 - Teste de médias e síntese da análise de variância (quadrado médio) da deposição de calda de pulverização ( $\mu 1 \mathrm{~cm}^{-2}$ ) no terço superior, médio inferior do dossel da cultura da soja e razão de deposição para os estandes de 160000 e 240000 plantas ha $^{-1}$ nos espaçamentos entrelinhas de 0,$30 ; 0,40 ; 0,50 ; 0,60 ; 0,70 \mathrm{~m}$ e $0,50 \mathrm{~m}$, em semeadura com linhas cruzadas.

\begin{tabular}{|c|c|c|c|c|}
\hline & Terço superior & Terço médio & Terço inferior & Razão de deposição \\
\hline \multicolumn{5}{|l|}{ Espaçamento } \\
\hline $0,30 \mathrm{~m}$ & $0,399 \mathrm{a}$ & $0,196 \mathrm{a}$ & $0,083 b$ & $5,30 \mathrm{~b}$ \\
\hline $0,40 \mathrm{~m}$ & $0,387 \mathrm{a}$ & $0,222 \mathrm{a}$ & $0,102 \mathrm{ab}$ & $3,99 \mathrm{ab}$ \\
\hline $0,50 \mathrm{~m}$ & $0,395 \mathrm{a}$ & $0,221 \mathrm{a}$ & $0,119 \mathrm{ab}$ & $3,82 \mathrm{ab}$ \\
\hline $0,5 \times 0,5$ & $0,375 \mathrm{a}$ & $0,192 \mathrm{a}$ & $0,149 \mathrm{a}$ & $2,83 \mathrm{a}$ \\
\hline $0,60 \mathrm{~m}$ & $0,355 \mathrm{a}$ & $0,205 \mathrm{a}$ & $0,114 \mathrm{ab}$ & $3,24 \mathrm{ab}$ \\
\hline $0,70 \mathrm{~m}$ & $0,392 \mathrm{a}$ & $0,222 \mathrm{a}$ & $0,132 \mathrm{ab}$ & 3,39ab \\
\hline \multicolumn{5}{|l|}{ Estande } \\
\hline 160000 plantas ha $^{-1}$ & $0,396 \mathrm{a}$ & $0,206 \mathrm{a}$ & $0,115 \mathrm{a}$ & $3,99 \mathrm{a}$ \\
\hline 240000 plantas $\mathrm{ha}^{-1}$ & $0,372 \mathrm{a}$ & $0,213 \mathrm{a}$ & $0,117 \mathrm{a}$ & $3,54 \mathrm{a}$ \\
\hline Estande & $0,006723^{\mathrm{NS}}$ & $0,000765^{\mathrm{NS}}$ & $0,000058^{\mathrm{NS}}$ & $2,428407^{\mathrm{NS}}$ \\
\hline Espaçamento & $0,002127^{\mathrm{NS}}$ & $0,001537^{\mathrm{NS}}$ & $0,004215 *$ & $5,953106^{*}$ \\
\hline Estande $\mathrm{x}$ Espaçamento & $0,000644^{\mathrm{NS}}$ & $0,004188^{\mathrm{NS}}$ & $0,001984^{\mathrm{NS}}$ & $2,879298^{\mathrm{NS}}$ \\
\hline Resíduo & 0,001644 & 0,002680 & 0,004469 & 2,086012 \\
\hline $\mathrm{CV}(\%)$ & 17,41 & 24,71 & 34,86 & 38,39 \\
\hline
\end{tabular}

Médias seguidas de mesma letra minúscula na coluna não diferem estatisticamente pelo teste de Tukey a 5\% de probabilidade. NS. não significativo $(\mathrm{P}>0,05)$; *Significativo a $5 \%$ de probabilidade pelo teste $\mathrm{F}$; $\mathrm{CV}$ : coeficiente de variação.

Ciência Rural, v.44, n.8, ago, 2014. 
interferência dos componentes de arquitetura de plantas na qualidade de aplicação.

Esses resultados indicam que, nos arranjos com espaçamento entrelinhas maiores, as folhas ficam melhor distribuídas ao longo da haste principal da planta, enquanto, nos espaçamentos reduzidos, a maioria das folhas está disposta na parte superior da planta em busca da maior quantidade de radiação solar, aumentando o efeito "guarda-chuva" durante as aplicações. Este foi possivelmente o fator que mais favoreceu a deposição do terço inferior. Desse modo, pode ser indicada a utilização desses arranjos espaciais maiores para aumentar o tempo de fechamento e/ou reduzir o fechamento total das entrelinhas da soja, com respaldo indicativo de melhor e maior controle das doenças de baixeiro com a aplicação de produtos fitossanitários. Contudo, as cultivares possuem características peculiares em relação à sua arquitetura e variam conforme a época de semeadura, condições ambientais, arranjo espacial, população e estádio de desenvolvimento, de forma que é necessária uma análise detalhada para cada cultivar em função destes fatores, para que seja possível adequar o arranjo espacial em benefício da qualidade de aplicação dos produtos fitossanitários.

A análise de variância mostrou diferença significativa para a produtividade de grãos da cultura

Tabela 3- Teste de média e síntese da análise de variância (quadrado médio) da produtividade agrícola $\left(\mathrm{Kg} \mathrm{ha}^{-1}\right)$ para cultura da soja nos estandes de 160000 e 240000 plantas ha ${ }^{-1}$ nos espaçamentos entrelinhas de 0,$30 ; 0,40$; 0,$50 ; 0,60 ; 0,70 \mathrm{~m}$ e $0,50 \mathrm{~m}$ e semeadura em linhas cruzadas $(0,5 \times 0,5) \mathrm{m}$ na safra $2011 / 2012$.

\begin{tabular}{ll}
\hline & Produtividade $\left(\mathrm{kg} \mathrm{ha}^{-1}\right)$ \\
\hline Espaçamento & $3196,0 \mathrm{ab}$ \\
$0,30 \mathrm{~m}$ & $3271,5 \mathrm{a}$ \\
$0,40 \mathrm{~m}$ & $3055,8 \mathrm{ab}$ \\
$0,50 \mathrm{~m}$ & $3001,8 \mathrm{~b}$ \\
$0,5 \mathrm{x} 0,5$ & $3025,8 \mathrm{ab}$ \\
$0,60 \mathrm{~m}$ & $3115,8 \mathrm{ab}$ \\
$0,70 \mathrm{~m}$ & \\
Estandes & $3121,2 \mathrm{a}$ \\
160000 plantas ha \\
240000 plantas ha \\
${ }^{-1}$ & $3100,9 \mathrm{a}$ \\
Estande & $4920,968^{\mathrm{NS}}$ \\
Espaçamento & $88428,888^{*}$ \\
Estande x Espaçamento & $6183,148^{\mathrm{NS}}$ \\
Resíduo & 26925,326 \\
Coeficiente de variação (\%) & 5,27 \\
\hline
\end{tabular}

Médias seguidas de mesma letra minúscula na coluna não diferem estatisticamente pelo teste de Tukey a $5 \%$ de probabilidade. ${ }^{\mathrm{NS}}$ : não significativo $(\mathrm{P}>0,05)$; $*$ Significativo a $5 \%$ de probabilidade pelo teste $\mathrm{F}$. da soja. A maior produtividade encontrada foi para o espaçamento de $0,4 \mathrm{~m}$ e a menor para a semeadura em linhas cruzadas.

De acordo com a EMBRAPA (2010), a cultivar 'BRS Valiosa RR', quando cultivada em altitude acima de $900 \mathrm{~m}$, deve ser semeada nos espaçamentos de $0,45 \mathrm{~m}$ ou $0,5 \mathrm{~m}$, com população de plantas entre 210000 a 250000 plantas ha ${ }^{-1}$. Destacase, neste trabalho, que a redução no estande em um terço não resultou em diferença significativa na produtividade, independentemente do espaçamento, indicativo da plasticidade da soja e de que é possível economizar com sementes, desde que se tenha uniformidade na sua distribuição.

No presente estudo, a semeadura em linhas cruzadas obteve a menor produtividade entre os arranjos testados. Resultados semelhantes a estes foram encontrados por BALBINOT JÚNIOR et al. (2012) e contrário por LIMA et al. (2012). Ressalva-se que os novos arranjos espaciais devem ser analisados com cautela, isto porque as cultivares respondem diferenciadamente aos arranjos (DUTRA et al., 2007). Com relação aos demais tratamentos, com semeadura somente em linhas paralelas em diferentes espaçamentos, não foram verificada diferenças estatísticas para o teste de média da produtividade (Tabela 3). Esses resultados concordam com MAUAD et al. (2010), HEIFFIG et al. (2006), KNEBEL et al.(2006), HEITHOLT et al. (2005), RAMBO et al. (2003) e RIGSBY \& BOARD (2003). Dessa forma, é possível afirmar que o potencial de rendimento da soja pode ser modificado pelo arranjo de plantas, sendo justificada a necessidade de estudos para determinar o melhor ajuste, mas com a ressalva de que o genótipo responde de forma diferenciada aos arranjos espaciais.

\section{CONCLUSÃO}

O arranjo espacial de plantas alterou a deposição no terço inferior, com maior depósito na soja semeada em linhas cruzadas, sendo $79,5 \%$ maior em relação ao obtido no espaçamento de $0,30 \mathrm{~m}$, bem como a distribuição de calda de pulverização quantificada pela razão de deposição. O espaçamento de $0,40 \mathrm{~m}$ proporcionou a maior produtividade e a semeadura em linhas cruzada a menor. Já a população de plantas não interferiu na deposição de calda de pulverização e produtividade da cultura.

\section{AGRADECIMENTOS}

Goiás - FAPEG
A Fundação de Amparo a Pesquisa do Estado de

Ciência Rural, v.44, n.8, ago, 2014. 


\section{REFERÊNCIAS}

BALBINOT JUNIOR, A.A. et.al. Avaliação do sistema de plantio cruzado da soja - cultivar de hábito determinado, Cuiabá, MT, 2012. In: CONGRESSO BRASILEIRO DE SOJA, 6., Soja: Integração Nacional e Desenvolvimento Sustentável. Anais... Brasília: EMBRAPA, 2012. 1 CD ROOM.

DEBORTOLI, M.P. et al. Espectro de gotas de pulverização e controle da ferrugem-asiática-da-soja em cultivares com diferentes arquiteturas de planta. Pesquisa Agropecuária Brasileira, Brasília, v.47, n.7, p.920-927, 2012. Disponível em: <http://dx.doi. org/10.1590/S0100-204X2012000700007>. Acesso em: 23 maio, 2013. doi:10.1590/S0100-204X2012000700007.

DUTRA, L.M.C. et al. População de plantas em soja. In. REUNIÃO DE PESQUISA DE SOJA DA REGIÃO SUL, 35. 2007, Santa Maria, RS. Anais... Santa Maria: Universidade Federal de Santa Maria, 2007. p.95.

EMPRESA BRASILEIRA DE PESQUISA AGROPECUÁRIA (EMBRAPA). Laboratório de Imagem. Softwares Gratuitos. AFSOFT. Disponível em: <http://www.cnpdia.embrapa.br/ labimagem/afsoft.php>. Acesso em: 13 fev. 2012.

EMPRESA BRASILEIRA DE PESQUISA AGROPECUÁRIA (EMBRAPA). Cultivares de soja Minas Gerais e Região Central do Brasil, safra 2010/2011. Londrina: Embrapa Soja, 2010. 49p.

FERREIRA, M.C.; OLIVEIRA, J.R.G. Aplicação de fungicidas na cultura da soja com pulverizador costal pressurizado e manual elétrico com bico rotativo para volume baixo. Parte 1: Cobertura. In: SIMPÓSIO INTERNACIONAL DE TECNOLOGIA DE APLICAÇÃO DE AGROTÓXICOS, 4., 2008, Ribeirão Preto, SP. Artigos... Campinas: Instituto Agronômico de Campinas, 2008. 1 CD ROM.

FERREIRA, D.F. Sisvar: a computer statistical analysis system. Ciência e Agrotecnologia, Lavras, v.35, n.6, p.10391042, 2011. Disponível em: <http://dx.doi.org/10.1590/ S1413-70542011000600001>. Acesso em: 23 mai. 2013. doi: $10.1590 / \mathrm{S} 1413-70542011000600001$

FERREIRA, M.C. Critérios para o início das aplicações de fungicida e desempenho de pontas de pulverização no controle da ferrugem asiática da soja. 2012. 105f. (Doutorado em Agronomia) - Universidade de Passo Fundo, Passo Fundo.

HEIFFIG, S.L. et al. Fechamento e índice de área foliar da cultura da soja em diferentes arranjos espaciais. Bragantia, Campinas, v.65, n.2, p.285-295, 2006. Disponível em: <http://dx.doi. org/10.1590/S0006-87052006000200010>. Acesso em: 23maio, 2013. doi:10.1590/S0006-87052006000200010.

HEITHOLT,J.J. et al. Planting configuration x cultivar effects on soybean production in low-yield environments. Crop Science, Madison, v.45, p.1800-1808. 2005. Disponível em: <http://dx.doi. org/10.2135/cropsci2004.0603>. Acesso em: 24maio, 2013. doi:10.2135/cropsci2004.0603.
LIMA, S.F. et al. Efeito da semeadura em linhas cruzadas sobre a produtividade de grãos e a severidade da ferrugem asiática da soja. Bioscience Journal, Uberlândia, v.28, n.6, p.954-962, 2012.

KNEBEL, J.L. et al. Influência do espaçamento e população de plantas sobre doenças de final de ciclo e oídio e caracteres agronômicos em soja. Revista Acta Scientiarum Agronomy, Maringá, v.28, n.3, p.385-392, 2006. Disponível em: <http://dx.doi. org/10.4025/actasciagron.v28i3.962>. Acesso em: 23 maio, 2013. doi:10.4025/actasciagron.v28i3.962.

MADALOSSO, M.G. et al. Cultivares, espaçamento entrelinhas e programas de aplicação de fungicidas no controle de Phakopsora pachyrhizi Sidow em soja. Ciência Rural, Santa Maria, v.40, n.11, p. 2256-2261, 2010. Disponível em: <http://dx.doi. org/10.1590/S0103-84782010001100002>. Acesso em: 23 maio, 2013.doi:10.1590/S0103-84782010001100002.

MAUAD, M. et al. Influência da densidade de semeadura sobre características agronômicas na cultura da soja. Revista Agrarian, Dourados, v.3, n.9, p.175-181, 2010.

MUNDSTOCK, C.M.; THOMAS, A.L. Soja: fatores que afetam o desenvolvimento e o rendimento. Porto Alegre: Evangraf, 2005. $31 \mathrm{p}$.

NATIONAL INSTITUTES OF MENTAL HEALTH (NIH). ImageJ. Disponível em: <http://rsb.info.nih.gov/ij/index.html $>$. Acesso em: 12 fev. 2012.

PALLADINI, L.A. et al. Choice of tracers for the evaluation of spray deposits. Scientia Agricola, Piracicaba, v.62, n.5, p.440445, 2005. Disponível em: < http://dx.doi.org/10.1590/S010390162005000500005>. Acesso em: 23 maio, 2013. doi:10.1590/ S0103-90162005000500005.

PHIPPS, P. et al. Asian soybean rust: frequently asked questions III: control with fungicides. Blacksburg:Virginia Cooperative Extension, Virginia Tech, 2006.p.450-303.

PIRES, J.L.F. et al. Efeito de populações e espaçamentos sobre o potencial de rendimento da soja durante a ontogenia. Pesquisa Agropecuária Brasileira, Brasília, v.35, n.8, p. 1541-1547, 2000. Disponível em: <http://dx.doi.org/10.1590/ S0100-204X2000000800006>. Acesso em: 23 maio, 2013. doi: 10.1590/S0100-204X2000000800006.

RAMBO, L. et al. Rendimento de grãos da soja em função do arranjo de plantas. Ciência Rural, Santa Maria, v.33, n.3, p.405-411, 2003. Disponível em: <http://dx.doi.org/10.1590/ S0103-84782003000300003>. Acesso em: 23 maio, 2013. doi:10.1590/S0103-84782003000300003.

RIGSBY, B.; BOARD, J.E. Identification of soybean cultivars that yield well at low plant populations. Crop Science, Madison, v.43, p.234-239. 2003. Disponível em: <http://dx.doi.org/10.2135/ cropsci2003.2340>. Acesso em: 24 maio, 2013. doi:10.2135/ cropsci2003.2340.

TAIZ, L.; ZEIGER, E. Fisiologia vegetal. Porto Alegre: Artmed, 2004. 719p. 